\title{
Towards an FBM Model Based Network Calculus Framework with Service Differentiation
}

\author{
Yu Cheng · Weihua Zhuang • Xinhua Ling
}

Published online: 22 April 2008

(C) Springer Science + Business Media, LLC 2008

\begin{abstract}
In this paper, we exploit traffic modeling with the fractional Brownian motion (FBM) process to develop a network calculus framework for end-toend performance analysis over a network provisioning differentiated services (DiffServ). The fundamental elements constituting the framework include accurate single-hop queueing analysis and three network calculuses that describe the stochastic behaviors when the traffic process is multiplexed, randomly split, or goes through a buffering system, respectively. Specifically, we develop a generic FBM based analysis for multiclass single-hop analysis where both inter-buffer priority and intra-buffer priority are used for service differentiation. Moreover, we present both theoretical and simulation studies to demonstrate that the output from the multiplexing, splitting, and buffering calculuses can still be modeled or well approximated by a properly parameterized FBM process. It is such preservation of the FBM characteristics that enables the concatenation of FBM based single-hop analysis into a network-wide performance analysis.
\end{abstract}

\footnotetext{
Y. Cheng $(\varangle)$

Department of Electrical and Computer Engineering, Illinois Institute of Technology, Chicago, IL, USA e-mail: cheng@iit.edu

W. Zhuang $\cdot$ X. Ling

Department of Electrical and Computer Engineering, University of Waterloo, Waterloo, ON, Canada

e-mail: wzhuang@uwaterloo.ca

X. Ling

e-mail: x2ling@uwaterloo.ca
}

Keywords network calculus - fractional Brownian motion - differentiated services $\cdot$ quality of service traffic model

\section{Introduction}

The current trend in service consolidation over Internet Protocol (IP) requires the best-effort service model of the legacy Internet to be enhanced to provide qualityof-service (QoS) guarantees, where the network performance analysis is of great importance.

The network calculus $[1,2]$ provides a theoretical framework for determining worst-case bounds on delay and buffering requirements in a network, under the condition that a traffic conditioning scheme (e.g., the leaky bucket) is applied at the source to force an arrival envelope. However, the framework takes a deterministic approach to provisioning services based on worstcase scenarios, which leads to a low resource utilization in networks aggregating a large number of random traffic processes. Moreover, the delay or loss incurred by the traffic conditioner (i.e., traffic conditioning effect) needs to be counted for an accurate end-to-end performance analysis, which adds extra complexity into the network calculus framework. The resource utilization issue motivates the recent efforts on stochastic network calculus $[3,4]$. Applying the stochastic network calculus incurs extra effort in designing the traffic conditioner to force a particularly selected arrival envelope, in addition to analyzing the traffic conditioning effect.

In this paper, we reinvestigate the network calculus framework by examining the fundamentals elements underpinning the end-to-end performance analysis. Our perspective is that an end-to-end performance 
analysis framework (equivalently, a network calculus framework) consists of four elements: the single-hop queueing analysis and three network calculuses that describe the stochastic behaviors when the traffic process is multiplexed, randomly split, or goes through a buffering system. The three calculuses are defined as multiplexing calculus, splitting calculus, and buffering calculus, respectively, which serve the objective of concatenating single-hop analysis into an end-to-end analysis. Applicability of a network calculus framework depends on the traffic modeling: the traffic model should facilitate both accurate single-hop analysis and determination of the output process from network calculuses. While the deterministic network calculus $[1,2]$ and stochastic network calculus [3, 4] resort to traffic conditioners to shape the incoming traffic for the convenience of end-to-end analysis, we instead focus on the direct traffic modeling approach to avoid the traffic conditioning impact on the performance.

The Markovian traffic model and associated queueing analysis have been studied extensively and intensively as a tool to evaluate the performance of voice or video applications [5, 6]. However, the Markovian model based analysis has the following limitations: (1) In high-speed networks, the loss probability in a finite buffer is hard to be obtained, which is often very conservatively approximated by the overflow probability in an infinite buffer system [5, 7]; (2) When a large number of Markovian sources are multiplexed, the large state space of the aggregate arrival process results in computational infeasibility; (3) End-to-end performance analysis requires accurately modeling the output process from a queue for next-hop analysis; the output process modeling of a Markovian input is also a difficult problem $[8,9]$; (4) Extensive network traffic measurement/analysis studies suggest that Internet traffic exhibits self-similar property or long-range dependence (LRD) [10,11], which can not be captured by the short-range dependent (SRD) Markovian model.

In this paper, the input traffic is modeled as a fractional Brownian motion (FBM) process [12]. In highspeed networks, the high degree of multiplexing justifies modeling the traffic aggregate as a Gaussian process according to the Central Limit Theorem [13]. The FBM process is a self-similar Gaussian process, which is therefore a suitable model for stochastic multiplexing analysis as well as for capturing the long-range dependence within the traffic.

With FBM modeling, both the overflow probability in an infinite buffer [13] and the loss probability in a finite buffer [7] can be accurately computed. In this paper, we generalize the FBM based queueing analysis to a multiclass environment where both inter-buffer priority and intra-buffer priority are used for service differentiation. Particularly, we develop accurate overflow/loss analysis techniques for a partitioned buffer system [14], which can provide different levels of loss protection within the buffer to implement an assured per-hop behavior (PHB) [15]. We also integrate the partitioned-buffer analysis with the FIFO buffer analysis through an inter-buffer priority scheme to achieve a complete performance analysis of a network node provisioning differentiated services.

It will be demonstrated that the stochastic properties of the FBM process retain well when the traffic undergoes the multiplexing, splitting, and buffering network calculuses. Specifically, we theoretically prove that the superposition of independent FBM processes still maintains or can be upbounded by an FBM process, depending on whether homogeneous or heterogeneous Hurst parameters are involved in the multiplexing. Moreover, the correlation structure of a traffic process is exactly preserved after a random split. In addition, we resort to theoretical analysis, intuitive reasoning, and simulation studies to investigate the buffer smoothing effect on a self-similar traffic process; we demonstrate that directly taking the FBM input process as the output process can give a reasonable accurate next-hop performance analysis, particularly when the buffer size is very small to support real-time data delivery, or quite large to guarantee a small loss probability. It is such preservation of the FBM characteristics that enables the concatenation of FBM based single-hop analysis into an accurate end-to-end performance analysis.

The remainder of this paper is organized as follows. Section 2 describes the system model. Section 3 presents the single-hop performance analysis based on FBM modeling. Section 4 investigates the network calculuses of multiplexing, splitting, and buffering. Section 5 presents numerical and simulation results to demonstrate the performance. Section 6 concludes this research.

\section{System model}

\subsection{DiffServ network}

Our objective is to develop an analytical framework for end-to-end performance analysis over a differentiated services network [16] as illustrated in Fig. 1. In the network, when traffic reaches a router at one of its input ports, packets within the traffic aggregate will be split into multiple streams and forwarded to different output ports for different destinations. At an output port, multiple traffic streams from different input ports 
Figure 1 Multiplexing, random splitting, and buffering in a differentiated services network

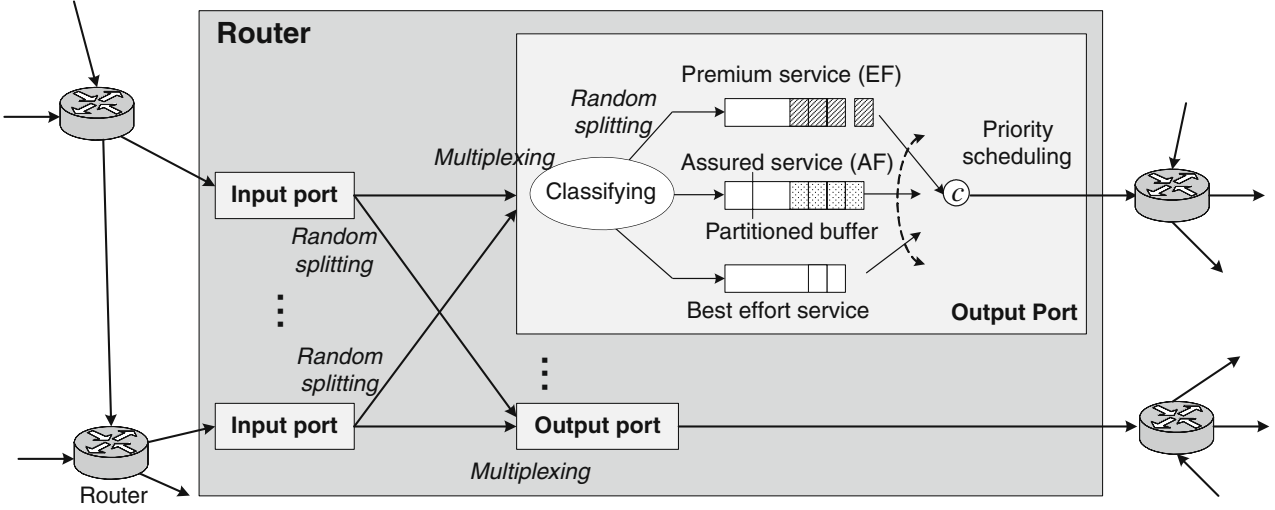

may be multiplexed and enter the same output buffer. In a DiffServ capable router, the output buffer is normally divided into multiple logic queues, served under a certain scheduling algorithm, to provision different classes of services. Thus, the traffic aggregate associated with an output port needs to be split again according to the DiffServ code point (DSCP) carried in each packet header [16] to generate the input process to the queue for each class.

A simple and efficient approach to differentiate services is to use a set of buffers served with priorities. There are two levels of priority. One is the inter-buffer priority (or priority queueing), where the traffic in a buffer of higher priority is served before that of lower priority. Typically, three buffers (served with high, medium, and low priority, respectively) are used at an output port to provision the two standard DiffServ perhop behaviors (PHBs), i.e. the expedited forwarding (EF) PHB characterizing the premium service [17] and the assured forwarding (AF) PHB characterizing the assured service [15], and the default best-effort service. The other level of priority, intra-buffer priority, is to serve traffic with a partitioned buffer [14], which provides different loss priorities while keeping the order of packets from the same traffic flow. The buffer for the assured service is usually a partitioned buffer.

In order to carry out the end-to-end performance analysis, two basic issues being addressed in this paper are accurate single-hop queueing analysis under the priority structure and proper traffic characterization under the effect of multiplexing, random splitting, and buffering. It is noteworthy that we assume the stochastic independence when we investigate the multiplexing and splitting effect: (1) The multiple arrival processes forwarded to an output port are considered independent; (2) A packet coming from an input port will be independently forwarded to one of the output ports at a certain probability, which defines the random splitting of an input process to the router. The random splitting is also applied when the aggregate process associated with an output port is further distributed into different service queues. The independence assumption is justified by the heterogenous, large-scale, high-multiplexing environment in the Internet, where the traffic streams along an Internet link normally consist of packets from different access networks as well as from various applications.

\subsection{LRD, self-similarity, and FBM}

Extensive measurements in recent years demonstrate that Internet traffic has the property of self-similarity or LRD $[10,11]$. A wide-sense stationary process $X(t)$ in discrete time ${ }^{1}$ is said to exhibit long-range dependence, if its autocorrelation function $r_{X}(k)$ decays with time lag $k$ taking the form

$r_{X}(k) \sim k^{-\gamma}, \quad$ as $k \rightarrow \infty$

where $0<\gamma<1$ and $f(k) \sim g(k)$ means $\lim _{k \rightarrow \infty} f(k) /$ $g(k)=a$, a nonzero constant. The Hurst parameter $H$ is commonly used to measure the degree of LRD, and is related to the parameter $\gamma$ in Eq. 1 by $H=1-\gamma / 2$.

Let the aggregated process $X^{(m)}=\left\{X_{k}^{(m)}\right\}$ be obtained by averaging the original traffic process $X$ over non-overlapping intervals, with each interval being $m$ time units in length. The autocorrelation function of $X^{(m)}$ follows $r_{X}^{(m)}(k) \sim k^{-\gamma}$, as $m \rightarrow \infty, k \rightarrow \infty$, meaning that the correlation structure of $X(t)$ is asymptotically preserved under the time aggregation. Thus, $X(t)$ is also defined to be asymptotically second-order selfsimilar (as-s). In fact, with $\frac{1}{2}<H<1$, as-s and LRD imply each other [11], and self-similarity and LRD are often used interchangeably in practice.

\footnotetext{
${ }^{1}$ In this paper, $X(t)$ is defined as the traffic volume, measured in packets or bits, arriving in the $t$ th time unit. We use $A(t)$ to denote the cumulative process indicating the total traffic volume from time 0 up to time $t$. $X(t)$ is also termed as the increment process of $A(t)$ as $X(t)=A(t)-A(t-1)$.
} 
There are other definitions of self-similarity. $X(t)$ is exactly second-order self-similar (es-s) if $r_{X}(k)=$ $\frac{\sigma^{2}}{2}\left[(k+1)^{2 H}-v 2 k^{2 H}+(k-1)^{2 H}\right]$, where $\sigma^{2}=\operatorname{var}[X(t)]$. The cumulative process of $X(t)$, denoted as $A(t)$, is distributional self-similar with Hurst parameter $H(\mathrm{H}-\mathrm{ss})$ if $A(t)={ }_{d} \alpha^{H} A(\alpha t)$ for all $\alpha>0$ and $t \geq 0$, where " $=d "$ denotes that the random process on both sides are equivalent in the sense of finite-dimensional distributions. The relationship between different levels of similarity are $[11,18]$

$\{\mathrm{H}$-ss $\} \subset\{$ es-s $\} \subset\{$ as-s $\}$.

In this paper, the FBM is used to model the cumulative input process $A(t)$. The standard (normalized) FBM process $\{Z(t): t \geq 0\}$ with Hurst parameter $H \in$ $[0.5,1)$ is a centered Gaussian process with stationary and ergodic increments which possesses the following properties [12]: (a) $Z(0)=0$, (b) $\operatorname{Var}\{Z(t)\}=t^{2 H}$, and (c) $Z(t)$ has continuous sample path. The FBM input $\{A(t): t \geq 0\}$ can be represented by

$A(t)=\lambda t+\sigma Z(t)$

where the mean arrival rate $E\{A(t) / t\}=\lambda$, and the variance $\operatorname{Var}\{A(t)\}=\sigma^{2} t^{2 H}$. Note that $\sigma^{2}$ is the variance of traffic in a time unit. FBM is an $\mathrm{H}$-ss process; when $0.5<H<1$, the FBM is both self-similar and long-range dependent.

\section{Single-hop analysis}

In this section, we present the FBM based overflow/loss analysis under the priority structure. Specifically, we consider that each output port uses a FIFO buffer and a partitioned buffer to provision the EF PHB and the AF PHB, respectively. The best-effort service is ignored without loss of generality. The overflow probability is defined as the ratio of the period that the queue length in the infinite buffer system spends above an indicated threshold to the total time. The loss probability is defined as the long-term ratio of the amount of the lost traffic to the amount of the total input traffic.

\subsection{FIFO buffer}

In a FIFO buffer with a stationary Gaussian input, the overflow probability can be accurately estimated by the maximum variance asymptotic (MVA) approach $[10,13]$. Let $\lambda$ denote the mean arrival rate, and $\sigma^{2}$ the variance of traffic in a unit time of the Gaussian input process. Let $\kappa=c-\lambda, X_{t}=A(t)-c t$, and $m_{x}$ the reciprocal of the maximum of $\sigma_{x, t}^{2}=\operatorname{Var}\left\{X_{t}\right\}$ / $(x+\kappa t)^{2}$ for a given $x$, i.e., $m_{x}=1 / \max _{t \geq 1} \sigma_{x, t}^{2}$. The MVA approximation of the overflow probability is then given by

$\mathbb{P}\left\{Q_{I}>x\right\} \approx \exp \left(-\frac{m_{x}}{2}\right)$

where $Q_{I}$ is used to denote the steady-state queue length in the infinite buffer system. For FBM input with parameters $\left(\lambda, \sigma^{2}, H\right), m_{x}$ can be explicitly computed by [10]

$m_{x}=\frac{4 \kappa^{\beta} x^{2-\beta}}{S \beta^{\beta}(2-\beta)^{2-\beta}}$

where $\beta=2 H$ and $S=\sigma^{2}$. The explicit expression of $m_{x}$ leads to the explicit expression of the overflow probability for the FBM input.

In [7], a simple method is proposed to estimate the loss probability $P_{L}(x)$ in a finite buffer system with buffer size $x$ from the overflow probability $\mathbb{P}\left\{Q_{I}>x\right\}$. By simulation studies and theoretical proof in the asymptotic case as $x \rightarrow \infty$, it is shown that

$P_{L}(x) \approx \alpha \mathbb{P}\left\{Q_{I}>x\right\}$

where $\alpha$ is a constant. With a Gaussian input, the constant $\alpha$ can also be explicitly calculated [7].

With the results in Eqs. 4-6, the packet loss probability for a finite buffer with an FBM input can be explicitly calculated. Extensive simulation results have been presented in [7], showing that the above overflow/loss calculation techniques give an accurate estimation of the overflow/loss probability for the entire buffer range, where $x$ can be set from a very small value to a very large value. The EF buffer, supporting the realtime multimedia applications, can particularly benefit from the accurate small buffer overflow/loss analysis.

\subsection{Partitioned buffer}

The AF PHB is provisioned by a partitioned buffer, where the input traffic includes $J(\geq 2)$ classes that have different loss probability requirements. The traffic admission policy is based upon a space reservation scheme, using the buffer partition vector $\boldsymbol{B}_{t}=\left(B_{1}\right.$, $B_{2}, \cdots, B_{J-1}$ ) to provide $J$ loss priorities, where 
$0=B_{0}<B_{1}<B_{2}<\cdots<B_{J-1}<B_{J}=B$ ( $B$ is the buffer size). Let $Q(t)$ be the amount of traffic queued in the buffer at time $t$. When $B_{j-1} \leq Q(t)<$ $B_{j} \quad(1 \leq j \leq J)$, only traffic of classes $\{j, j+1$, $\cdots, J\}$ is admitted into the buffer; traffic in the buffer is served according to the FIFO rule. With the partitioned buffer, the class- $J$ traffic is served with the highest priority and the smallest loss probability, while the class- 1 traffic the lowest priority and the largest loss probability.

We consider the total input process $A(t)$ including $J$ classes of traffic. Assume that all the traffic classes are independent and have the same Hurst parameter $H$. The class- $j$ input $A_{j}(t)$ is an FBM process characterized with $\lambda_{j}, \sigma_{j}^{2}$, and $H$. The number of class- $j$ packets arrived during $[0, t]$ is

$A_{j}(t)=\lambda_{j} t+\sigma_{j} Z_{j}(t), \quad 1 \leq j \leq J$.

In Section 4, we prove that the total input process $A(t)=\sum_{j=1}^{J} A_{j}(t)$ is also an FBM process with parameters of $\lambda=\sum_{j=1}^{J} \lambda_{j}, \sigma^{2}=\sum_{j=1}^{J} \sigma_{j}^{2}$, and $H$. We also prove that the multiclass FBM modeling in Eq. 7 is supported by the random splitting procedure. In practice, a self-similar video traffic process may be generated by the multi-layer coding, where a base layer contains the most important features of the video and some enhancement layers contain data refining the reconstructed video quality. If each video packet is randomly and independently associated with a layer with a certain probability, the layer-coded video traffic can then be modeled according to Eq. 7. With the AF PHB, different layers are marked to different classes for different levels of loss protection.

From the FBM based FIFO buffer analysis, we develop the overflow/loss calculation techniques for the partitioned buffer system with the multiclass FBM input. We resort to a localized steady state (LSS) assumption regarding the queueing behavior in a partition region (confined by two neighboring partition thresholds) that the steady-state overflow probability in a partition region can be determined by the initial status entering the region, the localized queueing behavior in the region (as in a separate buffer with the corresponding input), and the correlation within the input process characterized by the Hurst parameter $H$. Under the LSS assumption, we develop an iterative algorithm to calculate the overflow probabilities for all the $J$ classes and then obtain the loss probability for each class by exploiting the "loss versus overflow" mapping relationship between a finite size partitioned buffer and an infinite partitioned buffer.
Let $P_{V}(x)$ denote the overflow probability $\mathbb{P}\left\{Q_{I}>x\right\}$ and $P_{L}^{j}(1 \leq j \leq J)$ the loss probability for class- $j$, the overflow/loss calculation algorithm is:

Step 1: $\quad$ Set $P_{V}\left(B_{0}\right)=P_{V}(0)=1$

Step 2: $\quad$ for $j=1: J$

$$
\begin{aligned}
{[-\ln (} & \left.\left.P_{V}^{m, j}\left(B_{j}\right)\right)\right]^{\frac{1}{2-\beta_{j}}} \approx\left[-\ln \left(P_{V}\left(B_{j-1}\right)\right)\right]^{\frac{1}{2-\beta_{j}}} \\
& +z_{j}^{\frac{1}{2-\beta_{j}}}\left(B_{j}-B_{j-1}\right) ; \\
& \text { if } j \neq J \quad P_{L}^{j} \approx P_{V}\left(B_{j}\right) \approx \alpha_{j} P_{V}^{m, j}\left(B_{j}\right) ; \\
& \text { else } \quad P_{L}^{J} \approx \alpha_{J} P_{V}^{m, J}\left(B_{J}\right) ;
\end{aligned}
$$

end

In the algorithm, Eq. 8 indicates the overflow analysis according to the LSS assumption, where the localized queueing behavior in a partition region is characterized by Eq. 4 . In the $j$ th partition region, the input is an FBM process with parameters $\sum_{r=j}^{J} \lambda_{r}, \sum_{r=j}^{J} \sigma_{r}^{2}$ and $H$; with $\kappa_{j}=c-\sum_{r=j}^{J} \lambda_{r}$ and $S_{j}=\sum_{r=j}^{J} \sigma_{r}^{2}$, we have $z_{j}=$ $\frac{2 \kappa_{j}^{\beta}}{S_{j} \beta^{\beta}(2-\beta)^{2-\beta}}$. In the algorithm, the overflow probabilities in the infinite partitioned buffer are mapped from overflow probabilities (i.e., $\left.P_{V}^{m, j}\left(B_{j}\right)\right)$ in a promoted system, as indicated by Eq. 9, which is defined to facilitate the analysis [20]. When considering the loss probabilities in the finite partitioned buffer system, we find that the loss probabilities for class 1 to $J-1$ can be well approximated by the overflow probabilities $P_{V}\left(B_{1}\right)$ to $P_{V}\left(B_{J-1}\right)$, while the buffer truncation effect mainly applies to class $J$ as indicated by Eq. 10 . The theoretical details of the proposed partitioned buffer analysis and the calculation of the mapping factors $\alpha_{j}(1 \leq j \leq J)$ can be found in [20].

\subsection{Inter-buffer priority}

In the DiffServ router as shown in Fig. 1, the EF buffer has priority over the AF buffer and can access the full channel capacity $c$. The FIFO buffer analysis presented in Section 3.1 can be directly applied for EF PHB performance analysis.

On the other hand, the low-priority AF buffer can only access the channel after serving out all the EF traffic. The critical issue in the AF buffer analysis is to determine the leftover serving capacity. If the output process from the high-priority EF buffer is denoted as $D_{E F}(t)$, the serving capacity available to the AF buffer is $c-D_{E F}(t)$. However, it is difficult to accurately model the output process. We can see that the output 
process is upbounded by the arrival process. The studies in [21] demonstrate that using $c-A_{\mathrm{H} \text {-priority }}(t)$ as the leftover capacity provides a conservative performance analysis to the low-priority queue, with a reasonable accuracy in the large buffer regime in high-speed networks. In the next section, we show that using the input process as an approximation of the output process is particularly justified for self-similar traffic. Now consider the AF buffer with input $A_{A F}(t)$ and serving capacity $c-D_{E F}(t)\left(\approx c-A_{E F}(t)\right)$. The queueing system can be equivalently analyzed as a system having the capacity $c$ and the input $A_{A F}(t)+A_{E F}(t)$ [14].

\section{Network calculuses}

In this selection, we analyze the stochastic behaviors of the multiplexing, splitting, and buffering network calculuses for FBM traffic.

\subsection{Multiplexing calculus}

The input to the multiplexing calculus is multiple independent FBM processes, and the output is the superposition of the input processes. The superposition of selfsimilar processes has been investigated in [18]. For two independent es-s processes $X_{1}(t)$ with Hurst parameter $H_{1}$ and $X_{2}(t)$ with $H_{2}$, if $H_{1}=H_{2}=H, X_{1}(t)+X_{2}(t)$ is es-s with parameter $H$; if $H_{1} \neq H_{2}, X_{1}(t)+X_{2}(t)$ is as-s with parameter $\max \left(H_{1}, H_{2}\right)$. The FBM has a stronger self-similarity being $\mathrm{H}$-ss, for which we have the following proposition.

Proposition 1 For two independent FBM processes $A_{1}(t)$ with parameters $\left(\lambda_{1}, \sigma_{1}^{2}, H_{1}\right)$ and $A_{2}(t)$ with parameters $\left(\lambda_{2}, \sigma_{2}^{2}, H_{2}\right)$, if $H_{1}=H_{2}=H, A_{1}(t)+A_{2}(t)$ is an FBM process with parameters $\left(\lambda_{1}+\lambda_{2}, \sigma_{1}^{2}+\sigma_{2}^{2}, H\right)$; if $H_{1} \neq H_{2}, \quad A_{1}(t)+A_{2}(t)$ is as-s with parameter $\max \left(H_{1}, H_{2}\right)$.

Proof The effective bandwidth of a cumulative process $A(t)$ is defined as [22]

$E_{b}(s, t)=\frac{1}{s t} \log E\left[e^{s A(t)}\right] \quad 0<s, t<\infty$.

Particularly for an FBM process, the effective bandwidth is given by [22]

$E_{b}(s, t)=\lambda+\frac{s}{2} \sigma^{2} t^{2 H-1}$.
Let $E_{b, 1}(s, t), E_{b, 2}(s, t)$, and $E_{b, a}(s, t)$ denote the effective bandwidth of $A_{1}(t), A_{2}(t)$ and the aggregate $A(t)=$ $A_{1}(t)+A_{2}(t)$, respectively. Due to the independence between $A_{1}(t)$ and $A_{2}(t)$, we have

$$
\begin{aligned}
E_{b, a}(s, t) & =E_{b, 1}(s, t)+E_{b, 2}(s, t) \\
& =\left(\lambda_{1}+\lambda_{2}\right)+\frac{s}{2}\left(\sigma_{1}^{2}+\sigma_{2}^{2}\right) t^{2 H-1}
\end{aligned}
$$

when $H_{1}=H_{2}=H$. As a random traffic process can be uniquely characterized by its effective bandwidth, which is in the form of moment generating function (MGF), Eq. 13 indicates that $A(t)$ is an FBM process with parameters $\left(\lambda_{1}+\lambda_{2}, \sigma_{1}^{2}+\sigma_{2}^{2}, H\right)$.

When $H_{1} \neq H_{2}$, for example, $H_{1}<H_{2}$, we have

$$
\begin{aligned}
E_{b, a}(s, t) & =\left(\lambda_{1}+\frac{s}{2} \sigma_{1}^{2} t^{2 H_{1}-1}\right)+\left(\lambda_{2}+\frac{s}{2} \sigma_{2}^{2} t^{2 H_{2}-1}\right) \\
& <\left(\lambda_{1}+\lambda_{2}\right)+\frac{s}{2}\left(\sigma_{1}^{2}+\sigma_{2}^{2}\right) t^{2 H_{2}-1}
\end{aligned}
$$

which indicates $A(t)$ is not an FBM process, but its effective bandwidth is upbounded by that of the FBM process with parameters $\left(\lambda_{1}+\lambda_{2}, \sigma_{1}^{2}+\sigma_{2}^{2}, \max \left(H_{1}, H_{2}\right)\right)$. As $\{\mathrm{H}$-ss $\} \subset\{$ as-s $\}$, we have

$r_{X_{1}}(k) \sim k^{-\gamma_{1}}, r_{X_{2}}(k) \sim k^{-\gamma_{2}}, \quad$ as $k \rightarrow \infty$

where $\gamma_{1}=2-2 H_{1}, \gamma_{2}=2-2 H_{2}$, and $X(t)$ denotes the increment process. Due to the independence between $A_{1}(t)$ and $A_{2}(t)$, we can have the autocorrelation function $r_{X}(k)$ of the aggregate increment process $X(t)=X_{1}(t)+X_{2}(t)$ as

$r_{X}(k)=r_{X_{1}}(k)+r_{X_{2}}(k) \sim k^{-\gamma}, \quad$ as $k \rightarrow \infty$

where $\gamma=2-2 \max \left(H_{1}, H_{2}\right)$. Thus, the aggregate process $A(t)$ is as-s with parameter $\max \left(H_{1}, H_{2}\right)$.

Although the superposed process is not FBM when $H_{1} \neq H_{2}$, Eq. 14 indicates that taking the FBM process with parameters $\left(\lambda_{1}+\lambda_{2}, \sigma_{1}^{2}+\sigma_{2}^{2}, \max \left(H_{1}, H_{2}\right)\right)$ as an approximation can give a conservative performance analysis, which is a favorable property for QoS guarantee $[4,5,13]$. In addition, Proposition 1 can be readily applied when more than two FBM processes are aggregated.

\subsection{Splitting calculus}

An FBM process undergoing a splitting calculus will be randomly divided into multiple subprocesses. Specifically, we have the following proposition. 
Proposition 2 All the subprocesses generated by random splitting of the arrival process $X(t)$ maintain the same correlation structure as that of $X(t)$.

Proof Without loss of generality, we consider that the input process $X(t)$ is split into two subprocesses: each packet in $X(t)$ is assigned to subprocess $X_{1}(t)$ with probability $p$ and subprocess $X_{2}(t)$ with probability $1-p$. Let $V$ denote a Bernoulli random variable. ${ }^{2}$ With $X(t)$ indicating the number of packets arriving in the $t$ th time unit, the subprocess $X_{1}(t)=\sum_{i=1}^{X(t)} V_{i}$, where $V_{i}$ are independently and identically distributed (iid) Bernoulli random variables being independent of $X(t)$. If we denote $E[X(t)]=\lambda$ and $E[V]=v$, based on the independence assumptions, it is easy to derive by the probability generating function (PGF) technique [23] that

$E\left[X_{1}(t)\right]=E\left[\sum_{i=1}^{X(t)} V_{i}\right]=E[X(t)] E[V]=\lambda v$.

The autocovariance of $X_{1}(t)$ can be calculated as

$$
\begin{aligned}
r_{X_{1}}(k) & =E\left[X_{1}(t) X_{1}(t+k)\right]-\lambda^{2} v^{2} \\
& =E\left[\sum_{i=1}^{X(t)} V_{i} \cdot \sum_{i=1}^{X(t+k)} V_{i}\right]-\lambda^{2} v^{2} \\
& =E\left[X(t) X(t+k) E^{2}[V] \mid X(t), X(t+k)\right]-\lambda^{2} v^{2} \\
& =v^{2} r_{X}(k) .
\end{aligned}
$$

Similarly, $X_{2}(t)=\sum_{i=1}^{X(t)}\left(1-V_{i}\right)$, and we can get $r_{X_{2}}(k)=$ $(1-v)^{2} r_{X}(k)$.

Although Proposition 2 shows that the random splitting does not change the correlation structure, the marginal distribution of the subprocesses changes. Letting $G_{X}(z)$ and $G_{V}(z)$ denote the PGF of $X(t)$ and $V$, respectively, we have $G_{X_{1}}(z)=G_{X}\left(G_{V}(z)\right)$ [23]. By calculating $G_{X_{1}}(z)$, it can be seen that $X_{1}(t)$ is not Gaussian when $X(t)$ is Gaussian as the increment process of an FBM process. However, considering that each subprocess still consists of packets from a large number of traffic flows in a high-speed network, a Gaussian marginal distribution is a good approximation according to the Central Limit Theorem. While the mean of the subprocess is given by Eq. 17, its variance

\footnotetext{
${ }^{2}$ The Bernoulli random variable $V$ takes the values of 1 and 0 with the probability of $p$ and $1-p$, respectively, with mean value $E[V]=p$ and variance $\operatorname{var}[V]=p(1-p)$.
}

can also be determined by the PGF technique. For example, the variance of $X_{1}(t)$ can be computed as

$\operatorname{var}\left[X_{1}(t)\right]=E[X(t)] \operatorname{var}[V]+(E[V])^{2} \operatorname{var}[X(t)]$.

Summarizing the above discussions, the subprocess generated from randomly splitting an FBM process can be well approximated by another FBM process, parameterized according to Eqs. 17 and 18. Moreover, we can prove the following upbounding relationship.

Proposition 3 The effective bandwidth of a subprocess $X_{1}(t)$, which is generated by random splitting of an FBM arrival process $X(t)$, is upbounded by the effective bandwidth of the FBM process with parameters $\left(E\left[X_{1}(t)\right]\right.$, $\left.\operatorname{var}\left[X_{1}(t)\right], H\right)$.

Proof We follow the previous notations that each packet in $X(t)$ is independently assigned to subprocess $X_{1}(t)$ with probability $p$, and $V$ denotes the Bernoulli random variable with parameter $p$. The MGF of a cumulative process $A(t)$ is defined as $G(s)=E\left[e^{s A(t)}\right]$, which is interpreted as PGF when setting $z=e^{s}$. According to Eq. 11, we have $G(s)=e^{s t E_{b}(s, t)}$. Let $F(t)$ denote the FBM process with parameters $\left(E\left[X_{1}(t)\right]\right.$, $\left.\operatorname{var}\left[X_{1}(t)\right], H\right)$. In order to prove that the effective bandwidth of $X_{1}(t)$ is upbounded by that of $F(t)$, i.e., $E_{b, X_{1}}(s, t)<E_{b, F}(s, t)$, we equivalently need to prove that $\log G_{X_{1}}(s)<\log G_{F}(s)$.

With $X_{1}(t)=\sum_{i=1}^{X(t)} V_{i}$, it is not difficult to derive

$G_{X_{1}}(s)=G_{X}\left(\log G_{V}(s)\right)$

where $G_{V}(s)=p e^{s}+1-p$. Further more, in accordance with Eqs. 11 and 12, it can be seen that

$\log G_{X}(s)=s t \lambda+\frac{s^{2} t^{2 H}}{2} \sigma^{2}$.

Thus,

$$
\begin{aligned}
\log G_{X_{1}}(s) & =\log \left(G_{X}\left(\log G_{V}(s)\right)\right) \\
& =\log \left(G_{X}\left(\log \left(p e^{s}+1-p\right)\right)\right) \\
& =t \lambda \log \left(p e^{s}+1-p\right)+\frac{\sigma^{2} t^{2 H}}{2} \log ^{2}\left(p e^{s}+1-p\right)
\end{aligned}
$$

By applying the results of Eqs. 17 and 18 to Eq. 20, we can get

$\log G_{F}(s)=\operatorname{stp} \lambda+\frac{s^{2} t^{2 H}}{2}\left[\lambda p(1-p)+p^{2} \sigma^{2}\right]$.

Note that in a typical multiplexing scenario where the link capacity is much larger than the peak rate of 
a single source, the parameter $s$ affecting the effective bandwidth tends to $0[22,25]$. Therefore, we have

$e^{s p}-\left(p e^{s}+1-p\right) \approx 1+s p-(p(1+s)+1-p) \approx 0$.

Applying the result $p e^{s}+1-p \approx e^{s p}$ in Eq. 21, we can get

$\log G_{X_{1}}(s) \approx s t p \lambda+\frac{s^{2} t^{2 H}}{2} p^{2} \sigma^{2}$

and furthermore

$$
\begin{aligned}
\log G_{F}(s) & \approx \log G_{X_{1}}(s)+\frac{s^{2} t^{2 H}}{2}[\lambda p(1-p)] \\
& >\log G_{X_{1}}(s)
\end{aligned}
$$

The deviation between the actual effective bandwidth and the upper bound approximation is

$$
E_{b, F}(s, t)-E_{b, X_{1}}(s, t) \approx \frac{s t^{2 H-1}}{2}[\lambda p(1-p)]
$$

\subsection{Buffering calculus}

The buffering calculus describes the operation that the incoming traffic needs to wait in a buffer if the server is busy upon the arrival moment. Buffering normally will mitigate the burstiness of the incoming traffic; however, in the case that the input process is as-s or es-s with Hurst parameter $H$, it has been proved that the output process is as-s with the same Hurst parameter $H$ by both time-domain analysis [18] and frequency-domain analysis [24]. Furthermore, the studies in [19] show that the buffer smoothing effect takes place only for traffic with a small Hurst parameter by analyzing the inter-departure time distribution of the output process. The insensitivity of the self-similar traffic (particularly with a large Hurst parameter) to the buffer smoothing effect is also supported by the queueing analysis that $\log \mathbb{P}\left\{Q_{I}>x\right\} \sim x^{2-2 H}$, which indicates that buffering is not effective in reducing the overflow by smoothing out the burstiness $[10,11]$.

We can use intuitive reasoning to gain further insight into the buffer smoothing effect. Define a busy cycle as the time period between two consecutive time points at which the server changes from an idle state to a busy state. During a busy cycle, the amount of departures is equal to the amount of arrivals. If we construct the input process at a larger time scale, where the amount of arrivals in a busy cycle is considered as arrivals in a time unit and the busy cycle distribution is set as the interarrival time distribution, the departure process and the arrival process would be the same. This virtual construction procedure intuitively reveals that if the dominant time scale that determines the overflow behavior $[10,13]$ in the next-hop queue becomes larger than the average busy cycle in the upstream queue, the smoothing effect will gradually fade out.

On the other hand, the upstream buffering effect will not be obvious either when we consider next-hop overflow at a small queue length. It is noteworthy that the smoothing effect is in fact due to the truncation of the arrival process when the traffic load in a time unit exceeds the link capacity $c$. If the burstiness size leading to the overflow (at a small $x$ ) in the next-hop queue is smaller than the link capacity, the truncation effect or smoothing effect in the upstream will not take effect in this context.

In summary, the above discussions suggest that for self-similar traffic, directly taking the input process as an approximation of the output process can give a reasonably accurate next-hop performance analysis when the buffer size is very small to support real-time data delivery, or quite large to guarantee a small loss probability.

\section{Performance evaluation}

In this section, we present numerical analysis and simulation results to demonstrate the accuracy of the FBM based end-to-end analysis. The performance is demonstrated through three aspects: accuracy of the partitioned buffer analysis, validity of the output process approximation of the buffering calculus, and efficiency of the network calculus framework over a multi-hop, multi-path topology. In all the experiments, the FBM process is generated by a modified Random Midpoint Displacement (RMD) algorithm [26].

\subsection{Partitioned buffer analysis}

As the accuracy of FBM based overflow/loss analysis in a FIFO buffer has been examined in [7, 13], we here focus on examining the accuracy of the newly proposed partitioned buffer analysis. The MATLAB software is used to code and run the simulations for this experiment.

We consider a two-class FBM input served with a partitioned buffer of size 500. The class- 1 traffic is an FBM with $\lambda_{1}=10, \sigma_{1}^{2}=50$, and class- 2 an FBM with $\lambda_{2}=300, \sigma_{2}^{2}=100$. The Hurst parameter $H=0.8$ for both classes. The buffer is partitioned into two regions to differentiate the loss behaviors of class- 1 and 


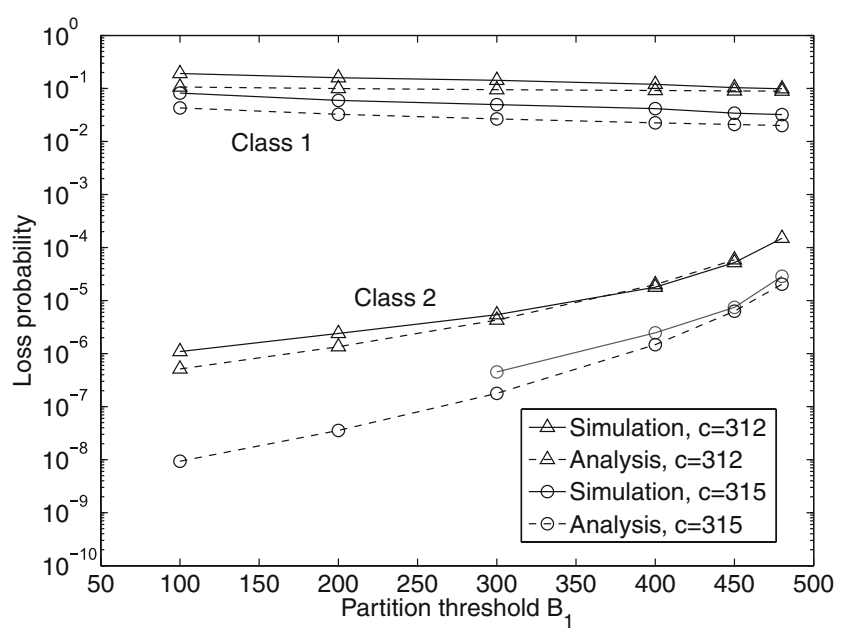

Figure 2 Loss probabilities for the two-class FBM input in a partitioned buffer

class-2, according to the admission policy described in Section 3.2. The units of mean arrival rate and channel capacity are packet/second, and the unit of buffer size is packet. Figure 2 shows the loss probabilities of two classes versus the partition threshold $B_{1}$, obtained from the analysis given in Section 3.2 and simulation. Furthermore, two scenarios are compared with $c=312$ and $c=315$. It is observed that the simulation results and the calculated loss probabilities are in a close match in both scenarios and with different partition configurations. It is also observed that for the self-similar FBM input, the loss probability is not very sensitive to the buffer space, while a small increase of the channel capacity can result in an obvious decrease of the loss probability.

\subsection{Output process approximation}

As we have rigorous theoretical propositions regarding FBM modeling under multiplexing and random splitting, here we use simulations to examine the validity of approximating the output process of a buffering calculus with the input FBM. The MATLAB software is used to code and run the simulations for this experiment.

We consider a two-hop scenario, where the first hop uses an infinite FIFO buffer with the link capacity $c=$ 83.3 packets/second (i.e., $c=1$ Mbps with packet size of 1,500 bytes) and the second hop use an infinite FIFO buffer with the link capacity of $0.95 c{ }^{3}$ We consider the infinite buffer system in order to investigate the

\footnotetext{
${ }^{3}$ The second-hop link capacity is set slightly smaller in order to observe overflow in the second-hop buffer in simulations, as the first-hop output rate is limited by the link capacity $c$.
}

upstream buffering effect on the next-hop queueing analysis at different time scales. The input FBM has parameters $\lambda=0.85 c, \sigma^{2}=0.75 \lambda$, and $H=0.77$. We use simulations to estimate the second-hop buffer overflow probability in two cases: (1) the input is the output process from the first-hop queue and (2) the input is approximated by the first-hop input (i.e. the first-hop output is approximated by the first-hop input). We also compute the overflow probability by the analytical techniques presented in Section 3.1. All the simulation results and analytical results are presented in Fig. 3.

Comparing the simulation results in the two cases as described, we can see that the overflow curve with the approximated input process is above the overflow curve with the real input process, giving a conservative performance estimation. In addition, the two overflow curves are very close when the queue length changes from a very small value 10 packets to a large value of 200 packets, which indicates that directly taking the input FBM as the output process is a reasonable approximation in the perspective of next-hop performance analysis. Moreover, it can be observed that the gap between the two curves is very small at both ends of the queue length range and reaches a maximum value in the middle. This observation justifies our intuitive reasoning presented in Section 4.3 that the smoothing effect grows at first along with the time scale, reaches the maximum point at the time scale approximately equal to the buffer busy cycle, and then gradually fades out when the time scale increases further. It is noteworthy that the analytical overflow curve with the input approximation also closely matches the simulation curves in a conservative manner, which shows that FBM based modeling and approximation is an appropriate tool for end-to-end performance analysis.

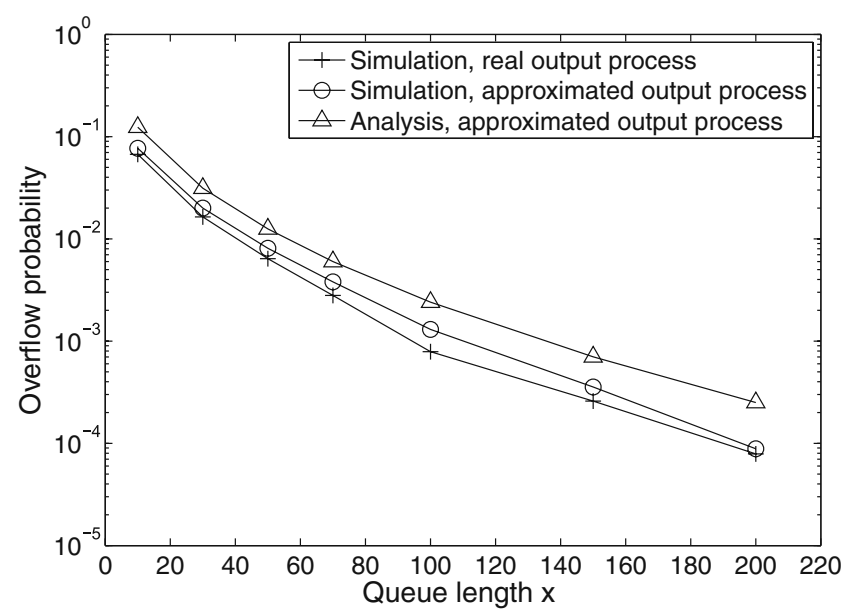

Figure 3 Overflow probability in the second-hop queue 
Figure 4 A multi-hop, multi-path topology for NS2 simulation

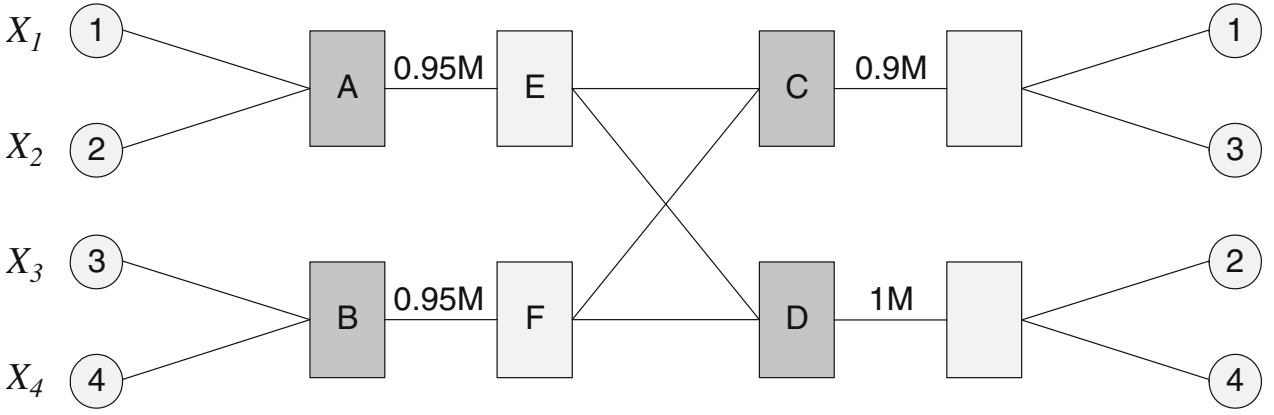

\subsection{Network-wide analysis}

In order to further demonstrate that the FBM model based framework is an efficient tool for end-to-end, and thus network-wide performance analysis, we use NS2 simulator [27] to simulate a multi-hop, multi-path topology as shown in Fig. 4, where the multiplexing, splitting, and buffering calculuses are involved to determine the packet loss probabilities at different network nodes.

In Fig. 4, we consider that four traffic flows transmit date from the source nodes to the corresponding destination nodes, following the shortest paths, respectively. The data traffic generated at each source node is considered as independent FBM process. The specific FBM parameters of the four traffic flows are listed in Table 1. All the network links have the capacity of 10 Mbps, except the four bottleneck links with respective capacities of $0.95,0.95,0.9$, and $1 \mathrm{Mpbs}$, as indicated. The packet size is 1500 bytes, and the buffer size at each network node is 50 packets. Based on the network topology and the given configuration, it can be seen that packet loss will happen only at nodes $\mathrm{A}, \mathrm{B}, \mathrm{C}$, and D.

To analytically obtain the packet loss probabilities at node A, B, C, and D, we need to apply the multiplexing calculus at nodes A, B, C, and D, and the splitting calculus at nodes E and F. Particularly, an packet output from $\mathrm{E}$ will go to node $C$ with probability 0.4 (flow 1 ), and to node $\mathrm{D}$ with probability 0.6 (flow 2 ); an packet

Table 1 Parameters of the FBM traffic flows

\begin{tabular}{llll}
\hline Traffic flow & $\lambda(\mathrm{Mbps})$ & $\sigma^{2}$ & $H$ \\
\hline$X_{1}$ & $0.4 \times 0.95$ & $0.75 \lambda$ & 0.8 \\
$X_{2}$ & $0.6 \times 0.95$ & $0.75 \lambda$ & 0.8 \\
$X_{3}$ & $0.5 \times 0.95$ & $0.75 \lambda$ & 0.8 \\
$X_{4}$ & $0.5 \times 0.95$ & $0.75 \lambda$ & 0.8 \\
\hline
\end{tabular}

output from $\mathrm{F}$ will go to node $C$ and $D$ with probability 0.5 (flow 3 ) and 0.5 (flow 4 ), respectively. The buffering calculus is applied at each node, so that we approximate the input traffic at each node as an FBM process. Moreover, finite buffer analysis is applied to obtain the packet loss probability.

NS2 simulation results and network calculus based analytical results are presented and compared in Fig. 5. We can observe that in the given small-buffer scenario, the network calculus framework has a robust performance: 1) In all the four network nodes under consideration, the analytical results and the simulation results are reasonably close, within one order of magnitude; 2) The deviations between the analytical and simulation results at different nodes maintain at the similar level; 3) The performance is stable through a wide loss range through $10^{-5}$ to $10^{-3}$. In addition, the results in Fig. 5 indeed demonstrate the conservativeness of the analytical results, due to the approximation taken in the splitting and buffering network calculuses.

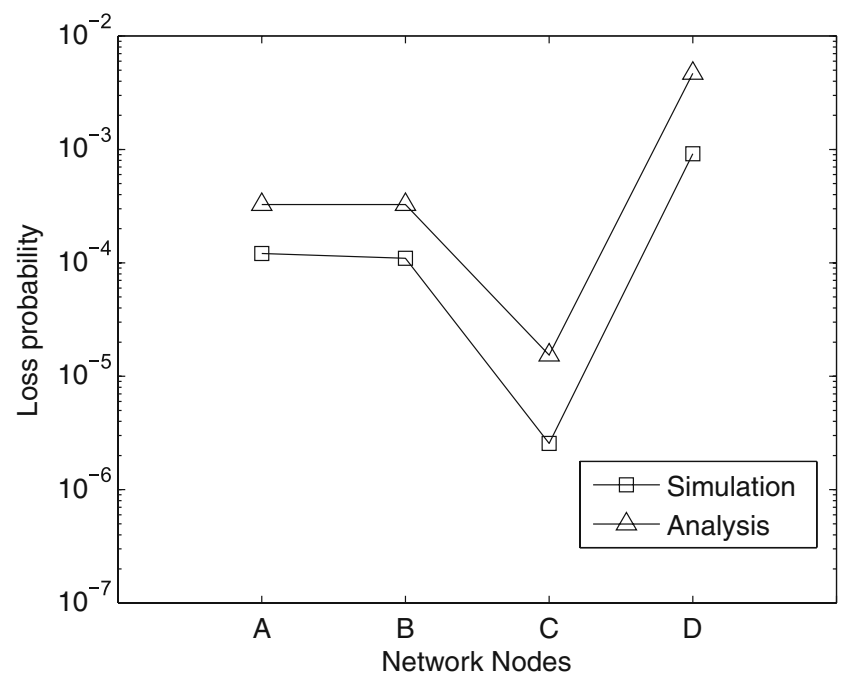

Figure 5 Packet loss probabilities at different network nodes 


\section{Conclusions}

In this paper, we present both theoretical and simulation studies to demonstrate that FBM based traffic modeling and associated queueing analysis enable the development of an efficient network calculus framework for end-to-end performance analysis over DiffServ networks. On one hand, we develop accurate FBM based overflow/loss analysis under the priority structure, where both inter-buffer and intra-buffer priorities are applied for service differentiation. On the other hand, we show that FBM based single-hop analysis can be concatenated into an accurate end-to-end performance analysis through multiplexing, splitting, and buffering network calculus operations. For future work, we will carry out further studies of the buffering calculus, where the accurate modeling of the output process is still an open issue. We also plan to further examine the FBM based network calculus framework against practical Internet traffic traces.

Acknowledgements The first author would like to thank his students Xiaohua Tian and Hongkun Li for their help in generating the NS2 simulation results.

\section{References}

1. Cruz RL (1991) A calculus for network delay, Parts I, II. IEEE Trans Inf Theory 37(1):114-141, January

2. Starobinski D, Karpovsky M, Zakrevski, LA (2003) Application of network calculus to general topologies using trun-prohibition. IEEE/ACM Trans Netw 11(3):411-421, June

3. Starobinski D, Sidi M (2000) Stochastically bounded burstiness for communication networks. IEEE Trans Inf Theory 46(1):206-212, January

4. Burchard A, Liebeherr J, Patek SD (2006) A min-plus calculus for end-to-end statistical service guarantees. IEEE Trans Inf Theory 52(9):4105-4114, September

5. Schwartz M (1996) Broadband integrated networks. Prentice Hall

6. Shroff NB, Schwartz M (1998) Improved loss calculations at an ATM multiplexer. IEEE/ACM Trans Netw 6(4):411-421, August

7. Kim HS, Shroff N (2001) Loss probability calculations and asymptotic analysis for finite buffer multiplexers. IEEE/ ACM Trans Netw 9(6):755-767, December

8. Takine T, Suda T, Hasegawa T (1995) Cell loss and output process analyses of a finite-buffer discrete-time ATM queueing system with correlated arrivals. IEEE Trans Commun 43(2):1022-1037, February/March/April

9. Elwalid A, Mitra D (1995) Analysis, approximation and admission control of a multi-service multiplexing system with priorities. In: Proc. IEEE INFOCOM'95, pp 463-472

10. Choe J, Shroff N (1999) Queueing analysis of high-speed multiplexers inculding long-range dependent arrival processes. In: Proc. IEEE INFOCOM'99, pp 617-624

11. Park K, Willinger W (2000) Self-similar network traffic: an overview. In: Park K, Willinger W (eds) Self-similar network traffic and performance evaluation, Wiley Interscience, pp 1-38

12. Norros I (1995) On the use of fractal Brownian motion in the theory of connectionless networks. IEEE J Sel Areas Commun 13(6):953-962, August

13. Choe J, Shroff N (1998) A central-limit-theorem-based approach for analyzing queue behavior in high-speed networks. IEEE/ACM Trans Netw 6(5):659-671, October

14. Cheng Y, Zhuang W (2003) Effective bandwidth of multiclass Markovian traffic sources and admission control with dynamic buffer partitioning. IEEE Trans Commun 51(9): 1524-1535, September

15. Heinanen J, Baker F, Weiss W, Wroclawski J (1999) Assured forwarding PHB group. IETF RFC 2597, June

16. Blake S, Black D, Carlson M, Davies E, Wang Z, Weiss W (1998) An architecture for differentiated services. IETF RFC 2475, December

17. Davie B, et al. (2002) An expedited forwarding PHB (perhop behavior). IETF RFC 3246, March

18. Song S, Ng JK-Y, Tang B (2004) Some results on the selfsimilar property in communication networks. IEEE Trans Commun 52(10):1636-1642, October

19. Ferng H-W, Chang J-F (2001) Characterization of the output of an ATM output buffer receiving self-similar traffic. In: Proc. IEEE GLOBECOM'01, pp 25-29

20. Cheng Y, Zhuang W, Wang L (2007) Calculation of loss probability in a finite size partitioned buffer for quantitative assured service. IEEE Trans Commun 55(9):1757-1771, September

21. Berger AW, Whitt W (1998) Effective bandwidths with priorities. IEEE/ACM Trans Netw 6(4):447-460, August

22. Kelly FP (1996) Notes on effective bandwidth. In: Kelly FP, Zachary S, Ziedins I (eds) Stochastic networks: theory and applications, Oxford, UK: Oxford Univ. Press, pp 141-168

23. Ross SM (2006) Introduction to probability models, 9th edn. Academic

24. Chan T-K, Li vOK (1998) Decomposition of network of queues with self-similar traffic. In: Proc. IEEE GLOBECOM'98, pp 3001-3006

25. Courcoubetis CA, Dimakis A, Stamoulis GD (2002) Traffic equivalence and substitution in a multiplexer with applications to dynamic available capacity estimation. IEEE/ACM Trans Netw 10(2):217-231, April

26. Norros I, Mannersalo P, Wang JL (1999) Simulation of fractional Brownian motion with conditional random displacement. Adv Perform Anal 2(1):77-101

27. The network simulator-NS2. Online: http://www.isi.edu/ nsnam/ns/ 


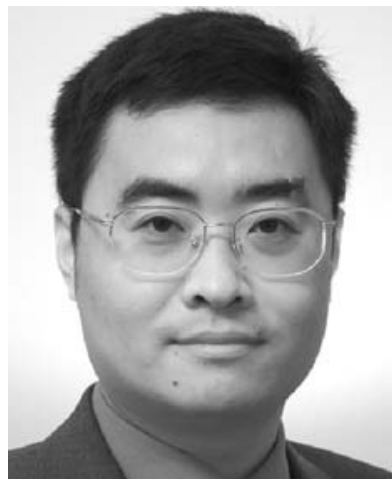

Yu Cheng received the B.E. and M.E. degrees in Electrical Engineering from Tsinghua University, China, in 1995 and 1998, respectively, and the Ph.D. degree in Electrical and Computer Engineering from the University of Waterloo, Canada, in 2003. From September 2004 to July 2006, he was a postdoctoral research fellow in the Department of Electrical and Computer Engineering, University of Toronto, Canada. Since August 2006, he has been with the Department of Electrical and Computer Engineering, Illinois Institute of Technology, USA, as an Assistant Professor. His research interests include application oriented networking, autonomic network management, Internet measurement and performance analysis, wireless networks, and wireless/wireline interworking. He received a Postdoctoral Fellowship Award from the Natural Sciences and Engineering Research Council of Canada (NSERC) in 2004, and a best paper award from the International Conference on Heterogeneous Networking for Quality, Reliability, Security and Robustness 2007 (QShine'07). He is an Associated Editor for IEEE Transactions on Vehicular Technology.

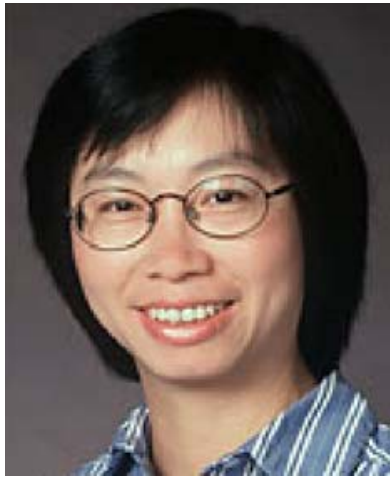

Weihua Zhuang received the B. Sc. and M. Sc. degrees from Dalian Maritime University, China, and the Ph.D. degree from the University of New Brunswick, Canada, all in electrical engineering. Since October 1993, she has been with the Department of Electrical and Computer Engineering, University of Waterloo, Canada, where she is a Professor. Dr. Zhuang is a co-author of the textbook Wireless Communications and Networking (Prentice Hall, 2003). Her current research interests include wireless communications and networks, and radio positioning. Dr. Zhuang is a co-recipient of a Best Paper Award from IEEE ICC 2007, a Best Student Paper Award from IEEE WCNC 2007, and the Best Paper Award from QShine 2007. She received the Outstanding Performance Award in 2005 and 2006 from the University of Waterloo and the Premier's Research Excellence Award
(PREA) in 2001 from the Ontario Government for demonstrated excellence of scientific and academic contributions. She is the Editor-in-Chief of IEEE Transactions on Vehicular Technology and an Editor of IEEE Transactions on Wireless Communications, EURASIP Journal on Wireless Communications and Networking, and International Journal of Sensor Networks. She is a Fellow of IEEE.

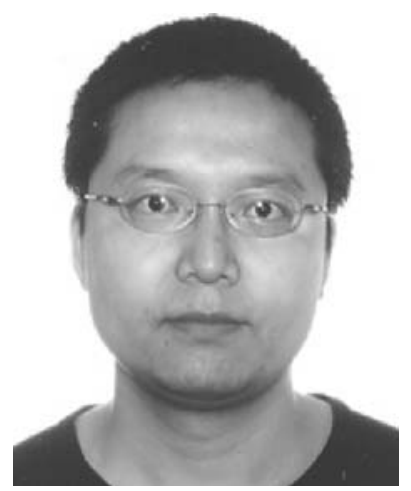

Xinhua Ling received the B.Eng. (1993) degree in Radio Engineering from Southeast University, China, the M.Eng. (2001) degree in Electrical Engineering from the National University of Singapore, Singapore, and the Ph.D. (2007) degree in Electrical and Computer Engineering from the University of Waterloo, Canada. Since November 2007 he has been a protocol analyst at Research In Motion (RIM), Waterloo, Canada. His research interests are in the area of cellular, WLAN, WPAN, ad hoc networks and their internetworking, including MAC and transport layer protocol design and performance analysis, and cross-layer design for Quality-of-Service support. 